NISTIR 7220

2005

\title{
HiCASS Target Tracking Sensor Study
}

\author{
for \\ Oceaneering Technology Corporation \\ Upper Marlboro, MD
}

Richard J. Norcross

Intelligent Systems Division

National Institute of Standards and Technology

Gaithersburg, MD 20899-8230 


\section{Disclaimer}

No approval or endorsement of any commercial product by the National Institute of Standards and Technology is intended or implied. Certain commercial equipment, instruments, or materials are identified in this report to facilitate understanding. Such identification does not imply a recommendation or endorsement by the National Institute of Standards and Technology, nor does it imply that the materials or equipment identified are necessarily the best available for the purpose.

\section{Copyright}

This publication was prepared by United States Government employees as part of their official duties and is, therefore, a work of the U.S. Government and not subject to copyright.

$\overline{\text { HiCASS Target Tracking Sensor Study }}$ 


\begin{abstract}
The High Capacity Alongside Sea base Sustainment (HiCASS) program intends to generate substantial improvements in skin-to-skin at-sea replenishment. HiCASS requires real-time tracking and dynamic load position sensing.
\end{abstract}

$\overline{\text { HiCASS Target Tracking Sensor Study }}$ 


\section{Table of Contents}

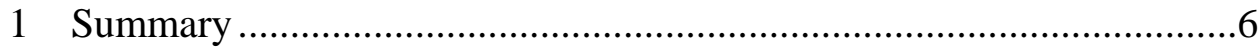

2 Introduction..................................................................................6

3 Methods, Assumptions, and Procedures ...................................................

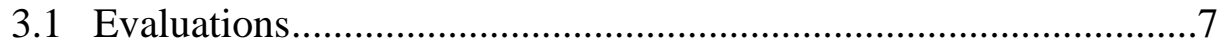

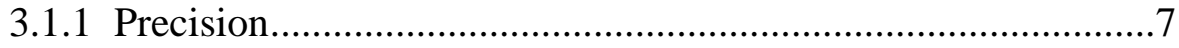

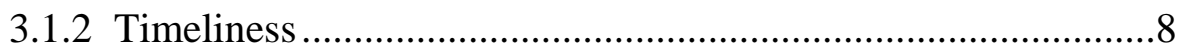

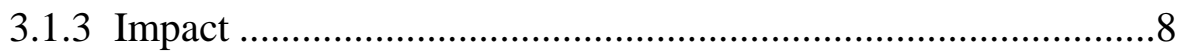

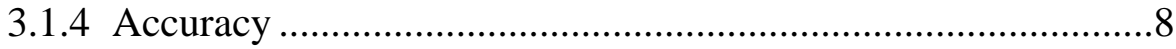

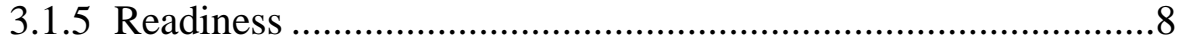

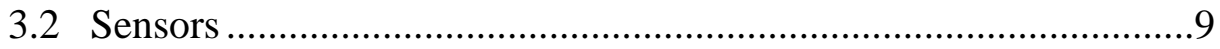

3.2.1 Ultra Wideband Radio Frequency Identification System ...........9

3.2.2 Three Dimensional Range Camera .............................................10

3.2.3 Panning Line Scan LADAR...................................................12

3.2.4 Fanning Laser Tracker ..........................................................13

3.2.5 Scanning Laser Tracker ............................................................13

4 Results and Discussions.................................................................13

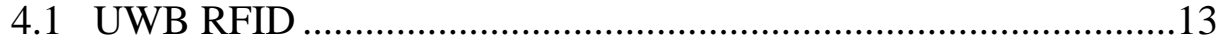

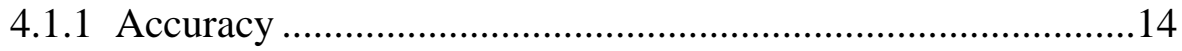

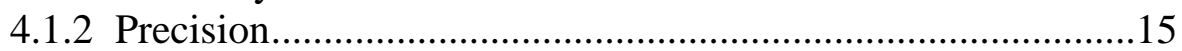

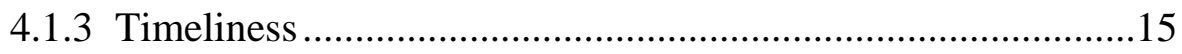

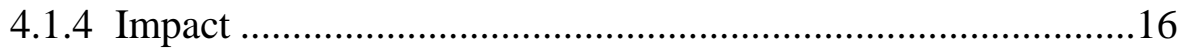

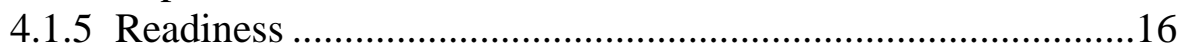

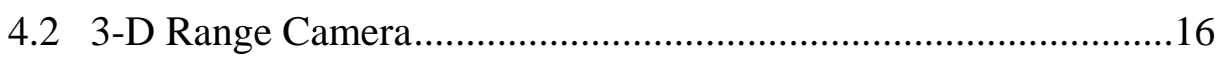

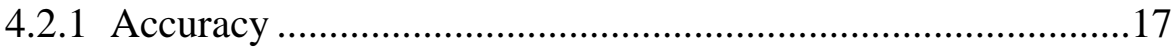

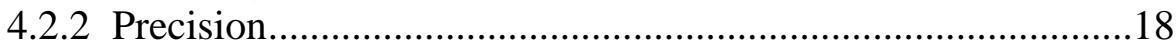

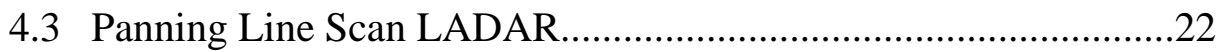

4.4 Fanning Laser Tracker .................................................................22

4.5 Scanning Laser Tracker ................................................................23

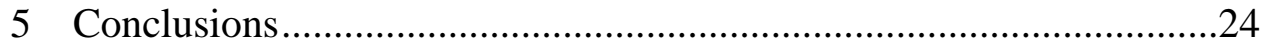

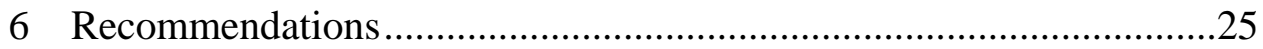

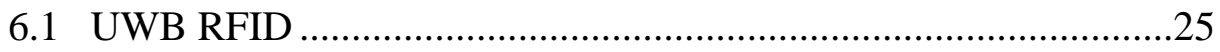

6.2 3-D Range Camera.......................................................................25

6.3 Panning Line Scan LADAR..........................................................25

6.4 Fanning Laser Tracker ..................................................................25

6.5 Scanning Laser Tracker .................................................................25

6.6 Vision...............................................Error! Bookmark not defined.

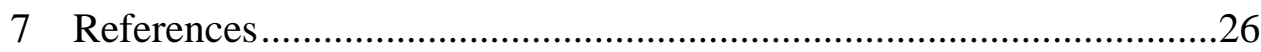

\section{List of Figures}

Figure 1. Sample Mean Position Error and Standard Deviation vs. Tag Height 15

Figure 2. Position Precision vs. Tag Repetition..........................................15

Figure 3. Lens Effect on Distance and Precision........................................17

HiCASS Target Tracking Sensor Study 
Figure 4. Lens Effect on Distance and Precision, Near Surface................17

Figure 5 Reading vs. Distance Correlation ............................................18

Figure 6 Prediction Interval vs. Distance................................................18

Figure 7 Prediction Interval vs. Surface Angle.......................................19

Figure 8. Corner Detection at $1.5 \mathrm{~m}$.....................................................20

Figure 9. Corner Detection at less then $0.5 \mathrm{~m}$......................................20

Figure 10. Corner Detection at $5 \mathrm{~m}$..................................................21

\section{List of Tables}

Table 1. UWB Tag Standard Deviation by Position and Height ...............14

Table 2. Comparison Summary .............................................................24

$\overline{\text { HiCASS Target Tracking Sensor Study }}$ 


\section{Summary}

The Expeditionary Logistics Future Naval Capability program intends to generate substantial improvements in the skin-to-skin variants of underway Connected Replenishment through the High Capacity Alongside Sea base Sustainment program (HiCASS). HiCASS requires solutions in many technologies including real-time tracking and dynamic load position sensing. The Intelligent Systems Division (ISD) of the National Institute of Standards and Technology (NIST) is investigating promising sensors for the HiCASS program. This paper reports an investigation on Ultra Wide Band Radio Frequency Identification tag.

\section{Introduction}

The Expeditionary Logistics Future Naval Capability program (ExLog FNC) identifies mature and evolving logistics technologies that, through focused investment, guidance and management, can provide enabling capabilities to the modern warfighter. The ExLog FNC intends to make a major impact on at-sea transfer of cargo through the High Capacity Alongside Sea Base Sustainment (HiCASS) program. HiCASS offers new approaches for fuel, cargo, vehicle and personnel transfer in a seaway while underway. It is intended to address both transfer between two large vessels and transfer from a large vessel to a small vessel.

The HiCASS program will develop innovative and integrated technology solutions built on existing naval systems. The typical naval Connected Replenishment (CONREP) method is the Standard Tensioned Replenishment Alongside Method (STREAM). The HiCASS program intends to generate substantial improvements to CONREP through skin-to-skin cargo transfer. HiCASS requires solutions in many technologies including:

- Real-time tracking/sensing of own ship/platform motions and those of the other ship/platform involved in the at-sea material transfer

- Motion compensating transfer systems capable of the precision required

- Distance Sensing

- Load Position/ Dynamics Sensing[1]

To fulfill the requirements in these and related technologies, HiCASS must develop and utilize appropriate sensory systems. The Intelligent Systems Division (ISD) of the National Institute of Standards and Technology (NIST) is investigating promising sensors for the HiCASS program. Ultra Wideband Radio Frequency Identification Tracking (UWB or UWB RFID) uses small transmitters mounted on the ISO container and an array of receivers mounted on the crane to determine the container position via triangulated differential arrival times. A Time-Of-Flight Range Camera (a.k.a. flash LADAR) uses the time-of-flight of reflected light to measure the distance to relatively close surfaces in a video field of view. A panning line-scan LADAR assembles a series of two dimension lines into a more accurate three dimension image. A LASER Tracker uses transmitters mounted on the crane and a receiver mounted on the container (or container ship) to determine position through angular relationships and the time-of-flight HiCASS Target Tracking Sensor Study 
os a single beam. This paper reports an investigations into these sensors and a comparison between them.

\section{Methods, Assumptions, and Procedures}

\subsection{Evaluations}

We use five criteria to evaluate the sensor system's ability to track ISO containers for HiCASS. The sensor data must be accurate, precise, and timely. The ideal sensor candidate also has minimum impact on operating procedures and is available in a hardened commercial package. Accuracy is the conformity of a measurement to the actual value. Precision is the degree of mutual agreement among a series of individual measurements. Timeliness is the time between periodic data sets. Impact is a subjective observation of additional burdens placed on the crew. A hardened commercial package is one designed for exterior use, preferably for harsh and maritime environments. We rank the criteria in order of significance as; precision, timeliness, impact, accuracy, and readiness.

\subsubsection{Precision}

This report emphasizes precision in its evaluations. While accuracy is a similar measure, accuracy can be corrected through calibration. Precision must be inherent in the sensor. NIST specifies the proper expression of uncertainty in TN 1297 [ref]. In this application, we concern ourselves with the precision of the sensor's next reading rather then the measurement of the last reading. Therefore, we express the sensor's precision with the magnitude of the prdiction interval.

The prediction interval is the sum of the mean and the product of the standard deviation of sample readings, the t-distribution for the desired confidence, and a function of the number of readings of the subsequent sample[9].

$$
P I=\mu \pm \sigma_{n} t_{n-1, A} \sqrt{1+1 / m}
$$

We use the size of the interval and a $0.95 \%$ confidence to compare precision.

$$
P=\sigma_{n} t_{n-1,0.95} \sqrt{1+1 / m}
$$

For example, when the standard deviation is taken from 25 readings, $n=25$. Our interest is in the accuracy of the next reading, so $m=1$. With a desired certainty of 0.95 the prediction interval is 2.9 times the standard deviation.

The ultimate goal for the HiCASS controller is to mate an ISO corner post with an ISO corner fitting. The post to fitting tolerance is $\pm 3 \mathrm{~cm}$. To ease the tolerance requirement, port crane spreader bars use fingers that slide along the ISO container and guide the post into the corner fitting. With guide fingers, the overall tolerance is up to $\pm 30 \mathrm{~cm}$. The combination of crane errors and the sensor errors must be less then this tolerance. We allocate one quarter of the tolerance for tracking sensor errors. Therefore the sensor must report position within $0.75 \mathrm{~cm}$ for mating without guide fingers and $7.5 \mathrm{~cm}$ for mating HiCASS Target Tracking Sensor Study 
with guide fingers. These values are subject to change based on developments of the control system.

\subsubsection{Timeliness}

Timeliness is the frequency of periodic data sets. Tracking sensors provide real-time positions in a dynamic environment. The position data must be sufficient to trace the target's motion and generate reasonable predictions of its future position. We use a goal of $20 \mathrm{~Hz}$ in our evaluation for HiCASS. Subsequent developments of the HiCASS control system may dictate a higher or lower frequency.

We base our evaluation of the sensor's data frequency on the manufacturer's claims. All sensors require additional computations to produce usable positions for HiCASS. We do not include these subsequent evaluations in the analysis. When the manufacturer claims a data frequency less then $20 \mathrm{~Hz}$, this report explores methods to enhance the output frequency.

\subsubsection{Impact}

Impact is a subjective evaluation of burdens imposed on the tracking procedure by the sensor. Ideally, sensor operation requires no specific activity or procedure. Minor and, in some cases, major impacts may be acceptable for HiCASS.

A minor impact limits HiCASS motions or requires extra motions. We consider spreader bar motion restrictions, such as to avoid obstructing the sensor, to be a minor impact. Likewise, the impact is minor when a sensor requires special manipulation by spreader bar to track the target.

Significant impact requires the cargo ship's crew to perform some duty. A major impact is a requirement for the cargo ship's crew to attach targets to the ISO container. Since the ship's crew must unlash containers and activate twist locks, a quick adjustment to a device on the ISO container is a minor impact.

\subsubsection{Accuracy}

Accuracy is the conformity a measurement to the actual value. Since proper calibration provides suitable accuracy, ease of calibration (particularly linearity) is a suitable measure for accuracy. We report detrimental impacts from minor environment changes as a loss of accuracy.

Any sensor that is repeatable, may be made accurate through calibration. Therefore we rank accuracy to be less important then precision, timeliness, and impact.

\subsubsection{Readiness}

Readiness is a subjective evaluation of the commercial status of the sensor. We feel HiCASS should not be dependent on significant sensor developments. The sensor's package indicates the maturity of the sensor system and reflects the sensor's reliability. A

$\overline{\text { HiCASS Target Tracking Sensor Study }}$ 
hardened commercial package is one designed for exterior use, preferably for harsh and maritime environments. Commercial packaging is not a requirement. The HiCASS developers may package any sensor to operate in a maritime environment. Therefore, readiness is the lowest priority criteria.

\subsection{Sensors}

This study reviewed five sensor types. Verifying experiments were conducted on some sensors, while other sensor evaluations rely on previous NIST investigations. Where appropriate, NIST investigations are supplemented with the manufacturer's claims.

\subsubsection{Ultra Wideband Radio Frequency Identification System}

An Ultra Wideband Radio Frequency Identification (UWB) system tracks embedded transmitters through their broadcast of short RF waveforms. Software solves for a signal source via the mathematical intersection of multiple hyperbolae as defined by the time difference of arrival (TDOA) of the signal at multiple receivers. The PAL650 Precision Asset Locator from Multispectral Solutions, Inc. represents the UWB tracking systems for this report. The UWB system consists of four (or more) passive Ultra Wide Band receivers, a collection of transmitters (a.k.a. tags) and a processing hub.

The UWB tags consist of a short pulse transmitter with a peak output power of approximately 0.25 Watts. Each tag periodically transmits a 72 pulse burst at $1 \mathrm{Mb} / \mathrm{s}$. The burst includes data that uniquely identifies the tag, synchronization fields, and control bits. The UWB system's bandwidth is $1.25 \mathrm{GHz}$. The bandwidth allows the signal to penetrate and circumvent obstacles. While the tags are asynchronous, the bursts are very short and collisions are infrequent.

The difference between the arrival time of two receivers defines a hyperbola of possible tag locations. A nonlinear optimization algorithm determines the 3-D tag coordinates from three or more hyperbolae. The receivers measure first time-of-arrival via a 100 $\mathrm{MHz}$ clock with a 10-tap digital delay. Since the equations use differential time of arrival and there are commonly 4 receivers generating 3 time differentials, the time quantization is approximately $300 \mathrm{pS}$.

UWB is a relatively low precision device. Improvements in timing, synchronization, search algorithms, and averaging may improve accuracy and precision. Timing is flexible based on the customer's requirements. Synchronization and algorithms are ongoing efforts of the manufacturer. NIST's effort evaluates accuracy and precision improvements through averaging. We estimate the effects of averaging through a Monte Carlo simulation. From the standard deviation of position in each of the major axes and random variables, software generates several thousand positions that are averaged and filtered to determine their relative impact on the output position.

The UWB system will have minor or no impact on the cargo ship. The Naval Facilities Engineering Service Center (Port Hueneme, CA) is evaluating UWB as a candidate in the ONR Naval Total Asset Visibility (NTAV) program[6]. If the Navy selects UWB

HiCASS Target Tracking Sensor Study 
tracking, all cargo will arrive at HiCASS with tags. However, HiCASS requirements are far greater then NTAV's and modifications will be required.

We make the precision and accuracy evaluations based on sample data. NIST uses UWB to track small robots in the Urban Search and Rescue test courses[7]. Researchers collect information on the tag's accuracy and precision through a series of static tests. We base the accuracy evaluation on the system's response to changes in the operating environment and the precision evaluation on a Monte Carlo simulation.

Accuracy, readiness, and frequency are evaluated through published accounts and antidotal experience of the PAL650 system. Accuracy is a function of the impact of noise and obstructions. Readiness reflects the level of the system hardening. Frequency is a function of the timing characteristics of the system. All five criteria are compared to other sensor options.

\subsubsection{Three Dimensional Range Camera}

The 3D range camera is a solid-state optical device for real-time three dimensional imaging. The range camera measures the phase relationship of reflected RF-modulated light at each pixel on a video array. The phase relationship correlates to the light's time of flight and measures the distance to a surface. The Swiss Ranger ${ }^{\mathrm{TM}} 2$ from CSEM SA [3] represents the 3D range camera systems for this report. The device generates intensity and distance values for each pixel of a $124 \times 160$ array. The 3D range camera is also known as a time-of-flight range camera and a flash ladar. We use these terms interchangeably.

Documented precision studies were not available for the Swiss Ranger ${ }^{\mathrm{TM}} 2$. Therefore, experimenters took extensive measurements with the range camera. We measured precision in regards to distance, angle, and as derived from the data image. These measurements quantified the camera's precision and provided understanding to the operation requirements.

Experimenters determine the precision as a function of the surface distance from data taken at several distances. Experimenters maintain the surface angle at each distance. A graphic representation shows the relationship between the prediction interval and the distance.

Experimenters determine the precision as a function of the surface angle by computing the prediction interval at several angles. Experimenters adjust the perpendicular distance to the surface such that the distance to the reflected point remains constant. A graphic representation shows the relationship between the prediction interval and the angle.

\subsubsection{Precision of Derived Position}

A range camera has sub-centimeter resolution but far less then centimeter precision. As will be shown in the Results section below, the prediction interval for each pixel exceeds the HiCASS tolerance requirements. However, there are over 22,000 pixels in each

HiCASS Target Tracking Sensor Study 
image. Detection algorithms filter the image to remove egregious noise, smooth data, extract prominent features and identify location.

A range camera is a relatively short-range device. Current RF modulation technology limits surface acquisition to $7.5 \mathrm{~m}$. Beyond this range, the detection suffers from insufficient returns and aliasing (e.g., an $8.5 \mathrm{~m}$ return is indistinguishable from a $1 \mathrm{~m}$ return). The range camera's optic Field Of View requires a range greater then $7.5 \mathrm{~m}$ to view a $6.1 \mathrm{~m}$ by $2.4 \mathrm{~m}$ ( $20 \mathrm{ft}$ by $8 \mathrm{ft}$ ) surface. Therefore, the observation of an entire ISO container by the flash ladar is impossible. Instead, the range camera identifies position from the detection of prominent features on the ISO container.

The most prominent ISO container features are the upper corner fittings. The typical corner is at the confluence of one vertical and two horizontal edges. The location of three corners will uniquely identify the position and orientation of the container. Since the range camera cannot view the entire ISO container, several range cameras need to identify separate features on the container. The experiments use a single range camera and the target is a single corner of an ISO container.

\subsection{Data Collection Procedure}

The experimenters compensated for some operation limitations of the test unit. The experimenters did not adjust collection parameters, but did adjust the lens and collected data in supportive environments. All collection positions were from the octant that is above, to the side, and in front of the ISO container to limit the subsequent data processing.

The Swiss Ranger ${ }^{\mathrm{TM}} 2$ has integration and threshold parameters to compensate for ambient light and surface reflectivity. Data is not currently available that identifies proper setting for given conditions and compilation of that data is beyond the scope of this effort. Therefore, this report reflects the values generated by the default settings.

The sample range camera performed poorly in daylight. Therefore, measurements of the ISO containers were collected after dark. The calibration and precision data measurements were taken indoors.

The range camera uses a manually adjusted lens. The lens position has a significant impact on the quality of the camera's intensity image and a smaller effect on the distance measurements. Lens position effects the focal length and the individual pixel vectors. For these experiments, operators adjust the lens for each data reading but use a single lens position for calibration readings.

The experimenter collects data from numerous distances from an ISO corner. All collection positions are from the octant that is above, to the side, and in front of the container. The experimenter moves the range camera , adjusts the lens to sharpen the intensity image, and collects images. Subsequent processing determines the corner position. The octant selection limits the number and complexity of required detection algorithms. A range camera implementation in HiCASS must function in all seven of the empty octants and will require several more detection algorithms.

HiCASS Target Tracking Sensor Study 


\subsection{Detection Algorithms}

The experimenters developed two algorithms to detect the ISO corner from a range image. The first interpretation software identifies the intersection of the container's two top edges. The second interpretation searches the image for the closest point, or nose. Software uses the second method when the first cannot find sufficient edges. HiCASS will require several algorithms and selection criteria in order to follow the ISO container in 3D space.

The primary interpretation algorithm identifies the ISO container's top edges and computes their intersection point. The algorithm inspects each vertical line for a convex edge. The slopes of linear reductions of the $5^{\text {th }}$ through $15^{\text {th }}$ pixel above and below each pixel determines the intersecting angle. The pixel with the greatest intersecting angle marks the convex edge. The algorithm collects a sequence of edges, estimates the corner, computes the lines before and after, and computes the intersection point. Although detecting the third edge into the corner would improve the results, the algorithm did not use the vertical edge.

The second interpretation algorithm searches the image for the closet point. This algorithm can only detect the corner from limited positions. The algorithm uses aggressive noise reduction and heavy filtering to smooth the image and clarify the closest point. As with the edge detection algorithm, the sensor must be above, outside, and in front of the container for the algorithm to function. The algorithm is useful when the ISO container is extremely close or when the container is extremely far away and accuracy requirements are relaxed.

Both algorithms post-process images saved in data files. For HiCASS, the interpretation algorithm will execute in real-time.

\subsubsection{Panning Line Scan LADAR}

Line Scan LADARs are popular in motion control systems. A single line scan generates a two dimensional map of the world in front of the sensor but cannot track a target in three dimensional space. Several tracking options derive from the line scan ladar. One option mounts the line scan ladar on a rotating axis. The axis steps across the scene as the ladar scans each line. This results in a three dimensional image with accuracy and precision of a line scan ladar. The LMS211 from Sick, Inc. is the representative line scan ladar for this report [3].

NIST is familiar with two panning line scan ladars, both based on the Sick LMS. The first mounts the Sick LMS along the axis of a stepper motor. The second reflects the laser with a tilting mirror. Both options are laboratory prototypes and both are relatively slow.

The Sick LMS has an absolute range limit of $80 \mathrm{~m}$ (260 ft). However, the system accuracy diminishes significantly in the long range mode. We use the short range mode (30 m, $98 \mathrm{ft}$ ) data for accuracy and precision evaluations.

The Sick LMS211 takes 101 readings per $13 \mathrm{~ms}$. The readings are every $1^{\circ}$ and the scan covers $100^{\circ}$ in front of the unit. The LMS211 begins a scan at either $50^{\circ}, 49.75^{\circ}, 49.5^{\circ}$,

$\overline{\text { HiCASS Target Tracking Sensor Study }}$ 
or $49.25^{\circ}$. Thus, the LMS211 can read distances at $1 / 4^{\circ}$ intervals in $53 \mathrm{~ms}$ and $1 / 2^{\circ}$ intervals in $26 \mathrm{~ms}$. We use the $1^{\circ}$ scan for timeliness comparisons.

\subsubsection{Fanning Laser Tracker}

The fanning laser tracker is a system of laser transmitters and active receivers. The transmitters send a fan of laser light through the operating environment. The fan's period identifies the transmitter to the receiver. The receiver measures the angles to the transmitters and computes the receiver's relative position from the difference between those angles. The fanning laser tracker derives positions for an unlimited number of receivers and automatically reacquires position after an interruption. The fanning laser tracker for this report is the Constellation 3D-I system from Arc Second, Inc. [4]

In this report, we use the manufacturer's data and supplement with other data where available. NIST researchers have experience with the Constellation system and we include their impressions. Most of the data is from a 2001 aircraft manufacturer's effort to measure the overall accuracy of the Constellation system.

The Constellation system began tests at sea in November 2004. NSWC-Carderock mounted the Constellation system on a T-ACS ship to monitor the motion of a crane boom. Test results are not yet available.

\subsubsection{Scanning Laser Tracker}

The scanning laser tracker scans a small area of the environment to track a highly reflective target. The target may be passive or active. High accuracy angular servos aim the LADAR transmitter at the target. A controller computes the target position from the angles and the LADAR distance. An active target derives the target's orientation from the incidence angles. We use the Laser Tracker II with the Smart Trak active target system by Automated Precision, Inc. for comparisons in this report[5].

We use manufacturer's data for all comparisons.

\section{Results and Discussions}

\subsection{UWB RFID}

The experiment's UWB system uses four receivers mounted in the corners of a $10 \mathrm{~m}$ by $20 \mathrm{~m}$ rectangle, 3 meters above the floor. The lateral spacing approximates a HiCASS implementation. The receivers' height reflects the available facility but is lower and flatter then desired. Since all of the receivers were at the same height, the results in the $\mathrm{Z}$ direction are worse then anticipated for HiCASS. However, since the receivers are closer, the $\mathrm{X}$ and $\mathrm{Y}$ direction results are better then would be anticipated for HiCASS.

Error! Reference source not found. lists the standard deviation for several hundred readings at numerous tag positions in NIST's USAR arenas. The test covers many

$\overline{\text { HiCASS Target Tracking Sensor Study }}$ 
locations within the arena and vertical shifts at those locations. We use the vertical shift data to evaluate accuracy and the standard deviation to evaluate precision.

Table 1. UWB Tag Standard Deviation by Position and Height

\begin{tabular}{|c|ccc|c|c|c|}
\hline Tag & $\mathrm{X}$ & $\mathrm{Y}$ & $\mathrm{Z}$ & & & \\
\hline 126 & 0.081 & 0.168 & 0.276 & & & \\
127 & 0.089 & 0.149 & 0.131 & & & \\
128 & 0.069 & 0.118 & 0.244 & & & \\
129 & 0.074 & 0.167 & 0.215 & & & \\
130 & 0.085 & 0.131 & 0.238 & & & \\
131 & 0.067 & 0.090 & 0.214 & & & \\
133 & 0.091 & 0.170 & 0.233 & & & \\
134 & 0.124 & 0.187 & 0.013 & & & \\
135 & 0.087 & 0.153 & 0.284 & & & \\
\hline
\end{tabular}

\subsubsection{Accuracy}

Figure 1 displays the mean position error and standard deviation as tags move away from the floor. The change in mean error with height shows the impact of environmental changes on reported position. Users correct a constant mean error through calibration. Since the change in mean error exceeds the HiCASS limit $(12 \mathrm{~cm}$ vs. $7.5 \mathrm{~cm})$, the UWB system does not have sufficient accuracy for HiCASS.

$\overline{\text { HiCASS Target Tracking Sensor Study }}$ 
Figure 1. Sample Mean Position Error and Standard Deviation vs. Tag Height
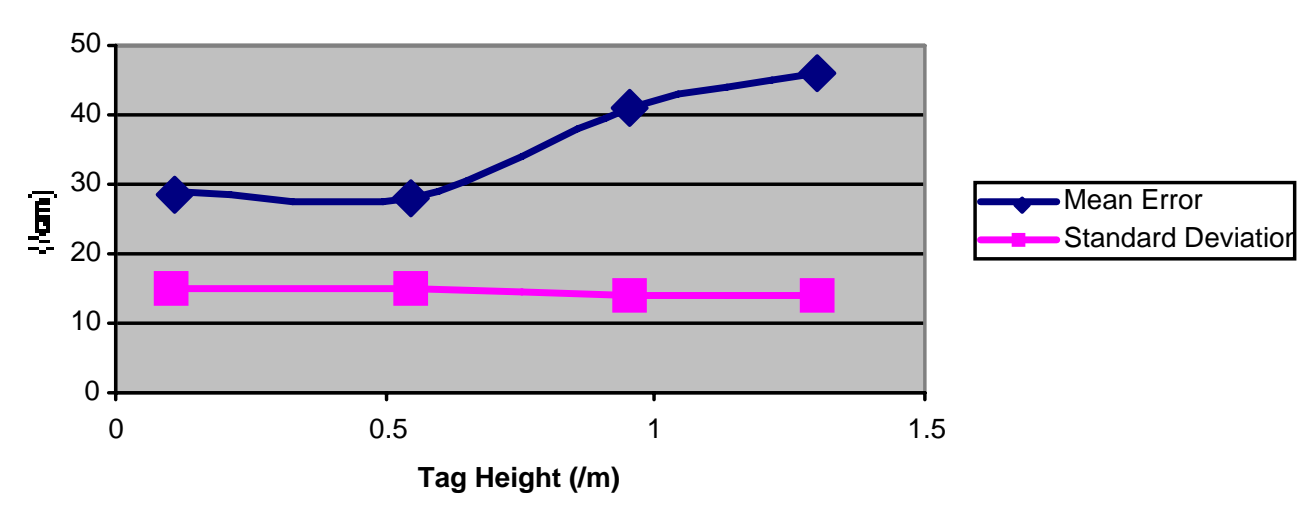

\subsubsection{Precision}

Figure 2 shows the relationship between precision and tag averaging as determined by the Monte Carlo simulation. 40 tags achieve the desired precision of $7.5 \mathrm{~cm}$. 40 tags may be 40 individual tags or one tag transmitting 40 times per sample. The current tags transmit once per second while the tag's maximum burst rate is $5000 \mathrm{~Hz}$ [2].

Figure 2. Position Precision vs. Tag Repetition

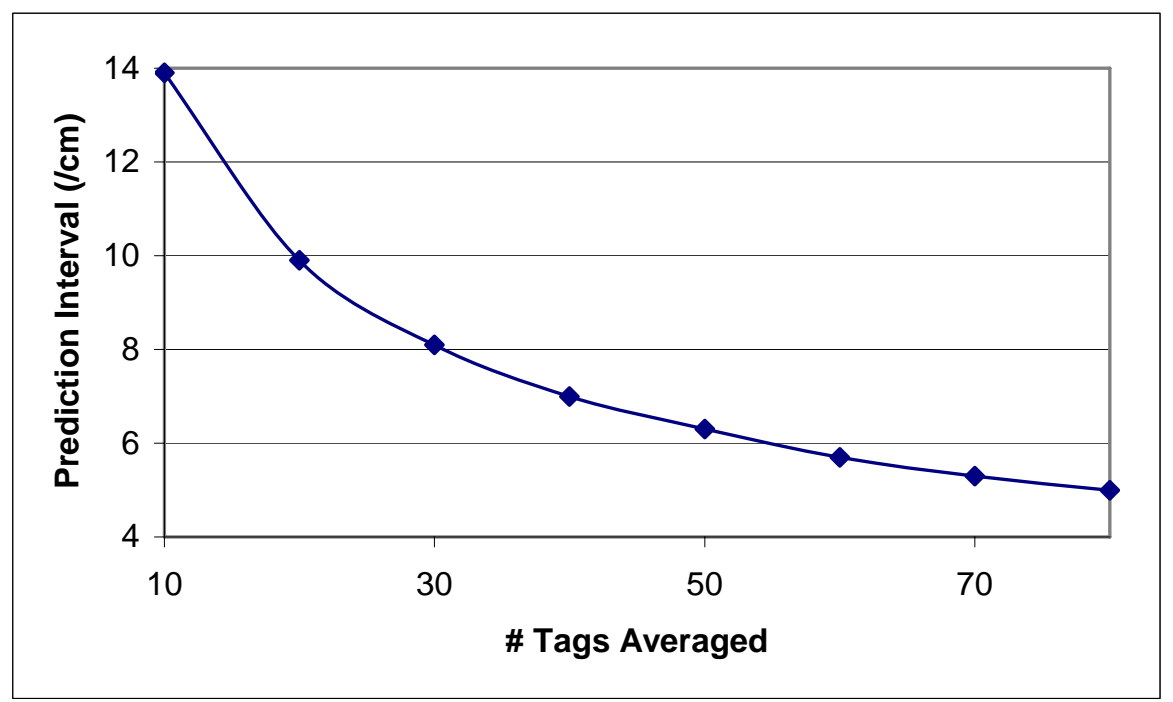

\subsubsection{Timeliness}

A coordinate system requires a minimum of three points to identify the origin and the primary axes. We envision each container will have three tag locations. Two containers will be active during tracking. The second provides the ship's motion when the target container is attached to the spreader bar.

$\overline{\text { HiCASS Target Tracking Sensor Study }}$ 
The maximum position rate is inverse of the product of the number of tags, the chirps per tag, the number of bits per chirp, and the duration of one bit. Therefore, the UWB system can generate 57 positions per second (1/(6 tags x 40 chirps/tag x 72 bits/tag x $1 \mu \mathrm{s} / \mathrm{bit})$ ). The HiCASS requirement of 20 positions per second requires a $35 \%$ duty cycle. This high duty cycle requires coordination between the transmitters to avoid interference. Therefore, the UWB system fulfills the HiCASS timeliness requirement, but may require substantial system modifications.

\subsubsection{Impact}

The UWB asset location system is a candidate for ONR's Naval Total Asset Visibility program. If UWB is selected, all DoD ISO containers will have identification tags. However the NTAV requires accuracy only within $1.6 \mathrm{~m}$ (5 ft) every few seconds [6]. HiCASS requires silence by most tags and 800 chirps per second by a few tags. The implementation requires the crew to change the tag mode when releasing or engaging the corner post twist locks. Therefore there would be minimal impact on the operations of the cargo ship's crew.

\subsubsection{Readiness}

Multi Spectrum Inc. manufactures the UWB system for interior applications. As described in previous sections, HiCASS requires significant enhancements. The UWB system is not ready for deployment.

\subsection{3-D Range Camera}

The 3-D range camera has adjustable thresholds, saturation limits and a mechanical lens. The thresholds and saturation limits correct data for environmental conditions and surface reflectivity. The lens focuses the image onto the pixel array. Experimenters did not adjust the image thresholds or saturation limits in the range camera's software. However, the camera focus had significant impact on close-in images. Since the HiCASS application requires close in observations, experimenters adjusted the camera lens to improve focus.

Figure 3 displays data collected from a stationary range camera $1.5 \mathrm{~m}$ from a perpendicular surface. The data shows the effect of the lens focus on the distance readings and the prediction interval of individual pixels. Clearly the operator desires the lens to be in the full CCW position to obtain the tightest prediction interval.

$\overline{\text { HiCASS Target Tracking Sensor Study }}$ 
Figure 3. Lens Effect on Distance and Precision

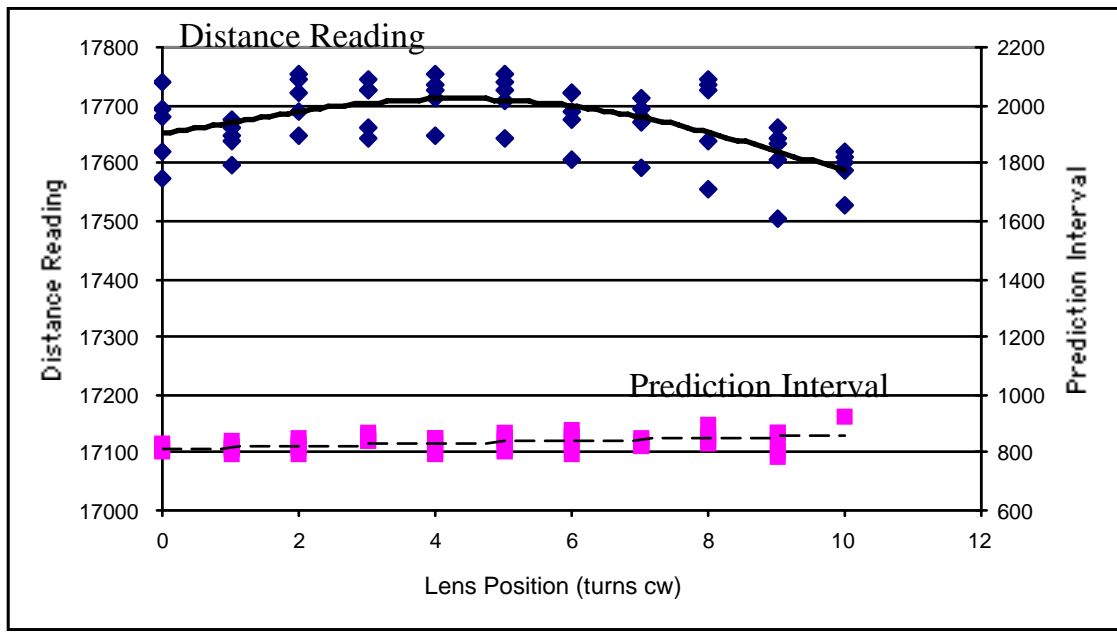

Figure 4 displays the same type of data collected from the same camera at roughly one half the distance from the surface. When the lens is fully CCW, the image is unusable. A useful image requires several turns of the lens.

Figure 4. Lens Effect on Distance and Precision, Near Surface

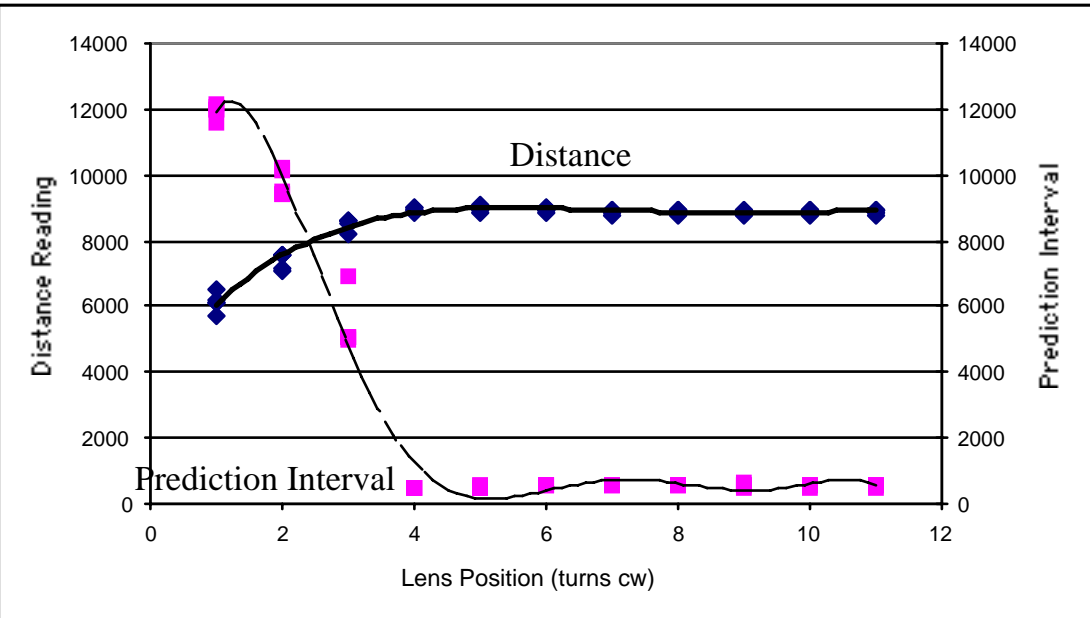

The HiCASS application requires the range camera to operate at various distances including, and arguably most importantly, very close. Therefore, the operators adjust the lens to provide the clearest image for the ISO identification experiments. Since the lens adjustment effects the focal length and, thereby, the pixel unit vectors, subsequent evaluation uses the range camera's distance precision not absolute position accuracy.

\subsubsection{Accuracy}

Users must convert the range camera's integer distance measures into engineering unit values. The experimenters collect data at several positions from a surface and correlate the readings with the distance. Since the optical focus impacts the data at close ranges

HiCASS Target Tracking Sensor Study 
and the experimenters are not able to directly measure the focal length, the data positions are at medium to long ranges and the focal length is constant throughout the measurements.

The data in Figure 5 has a linear coefficient is $0.000114 \mathrm{~m} / \mathrm{bit}$ with a $-0.365 \mathrm{~m}$ offset. Subsequent evaluations in this report use these coefficients even with images from different focal lengths. The coefficient of determination is 0.998 indicating a highly linear relationship. The 3-D range camera has sufficient linearity to meet the HiCASS accuracy requirement.

Figure 5. Reading vs. Distance Correlation

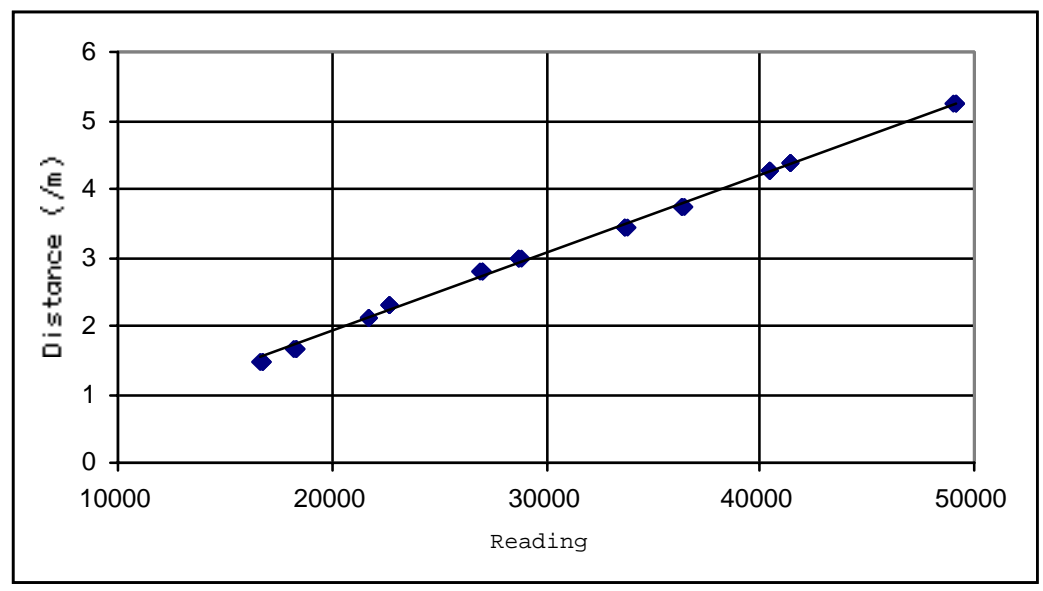

\subsubsection{Precision}

Figure 6 shows the prediction interval as a function of distance. While there appears to be a slight positive correlation, the coefficient of determination is 0.06 and the relationship is not significant. Figure 6 also shows that while the prediction interval of individual pixels is not a function of the target distance, it is also obviously too large for the application to utilize single pixel ranges. The individual pixels have a prediction interval of $15 \mathrm{~cm}$ while the HiCASS has a $7.5 \mathrm{~cm}$ maximum tolerance.

Figure 6. Prediction Interval vs. Distance

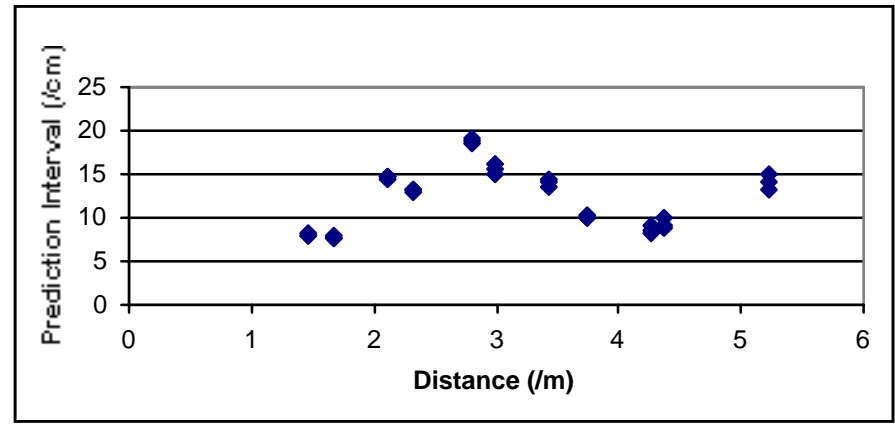

$\overline{\text { HiCASS Target Tracking Sensor Study }}$ 
Figure 7 shows the relationship between the prediction interval and the surface angle. The distance between the range camera and the image center is $2 \mathrm{~m}$ (79 in) for each data set. The results clearly show the prediction interval increases as the surface angle increases.

Figure 7. Prediction Interval vs. Surface Angle

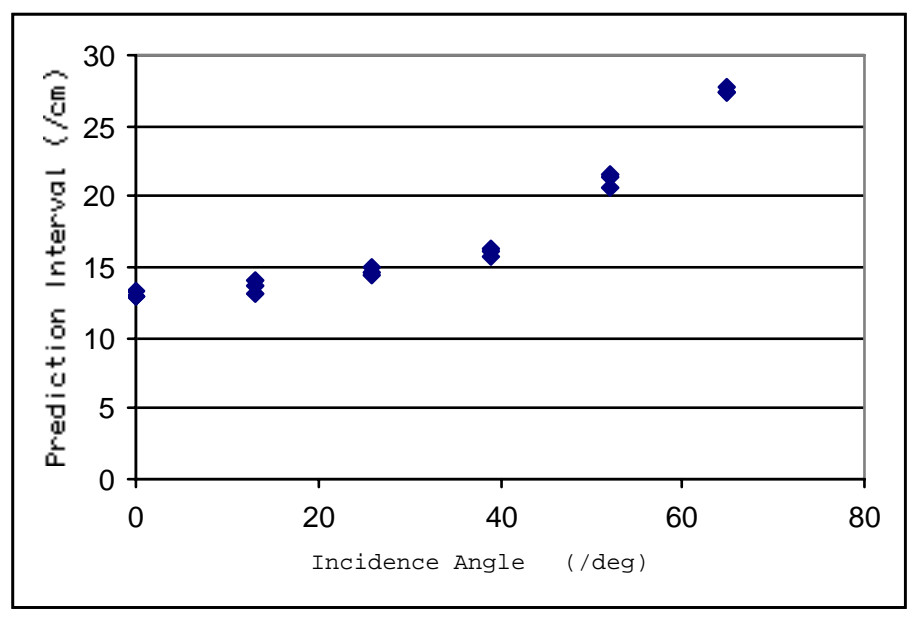

The prediction interval test results indicate the individual pixels of the range camera do not have the required precision for HiCASS. However, the range camera's image contains over 22,000 pixels. The range camera can achieve the required precision through filtering and evaluating the entire image.

\subsubsection{Target Detection}

The target to be tracked is the corner fitting of the ISO container. For these evaluations, we view the target from the opposite octant (above, to the side, and above the container). Figure 8 through Figure 10 show examples of the intensity and distance data from the range camera at different distances. Each figure shows the intensity data on the left and the distance data on the right. The images display significant noise in the distance measures particularly around the ground clutter to the right of the ISO container. The detection algorithm marks the convex edges with small blue squares and the target corner with a larger white square.

Figure 8 shows a typical image from approximately $1.5 \mathrm{~m}$. The intensity image is faint, but recognizable. The detected points on the two horizontal edges on the distance image are highlighted. The ground clutter noise in front and to the right of the container is prominent. The algorithm can easily evaluate and discard clutter noise.

$\overline{\text { HiCASS Target Tracking Sensor Study }}$ 
Figure 8. Corner Detection at $1.5 \mathrm{~m}$

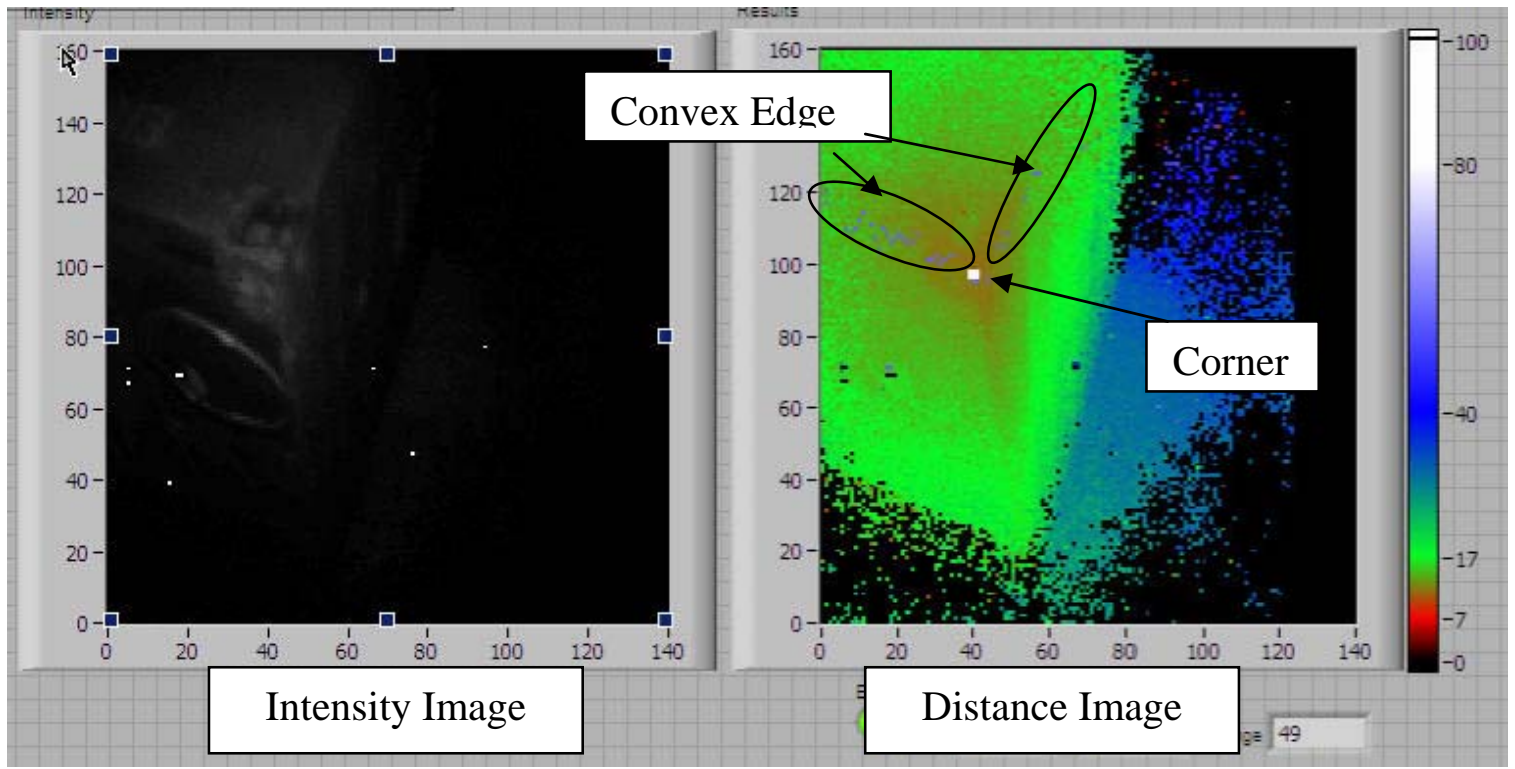

Figure 9 shows a typical image from less then one half meter. The container's corner fitting is very clear in both the intensity and distance images. The intensity image is very bright with some areas near the center washed out. The range camera cannot derive distances from the washed out areas as shown by the equivalent blacked out areas in the distance image. The detection algorithm found the container corner through the closest point algorithm (note lack of light blue squares on distance image). Figure 9 represents the current practical detection limit of the range camera. However, proper application of saturation limits may extend the range even closer.

Figure 9. Corner Detection at less then $0.5 \mathrm{~m}$

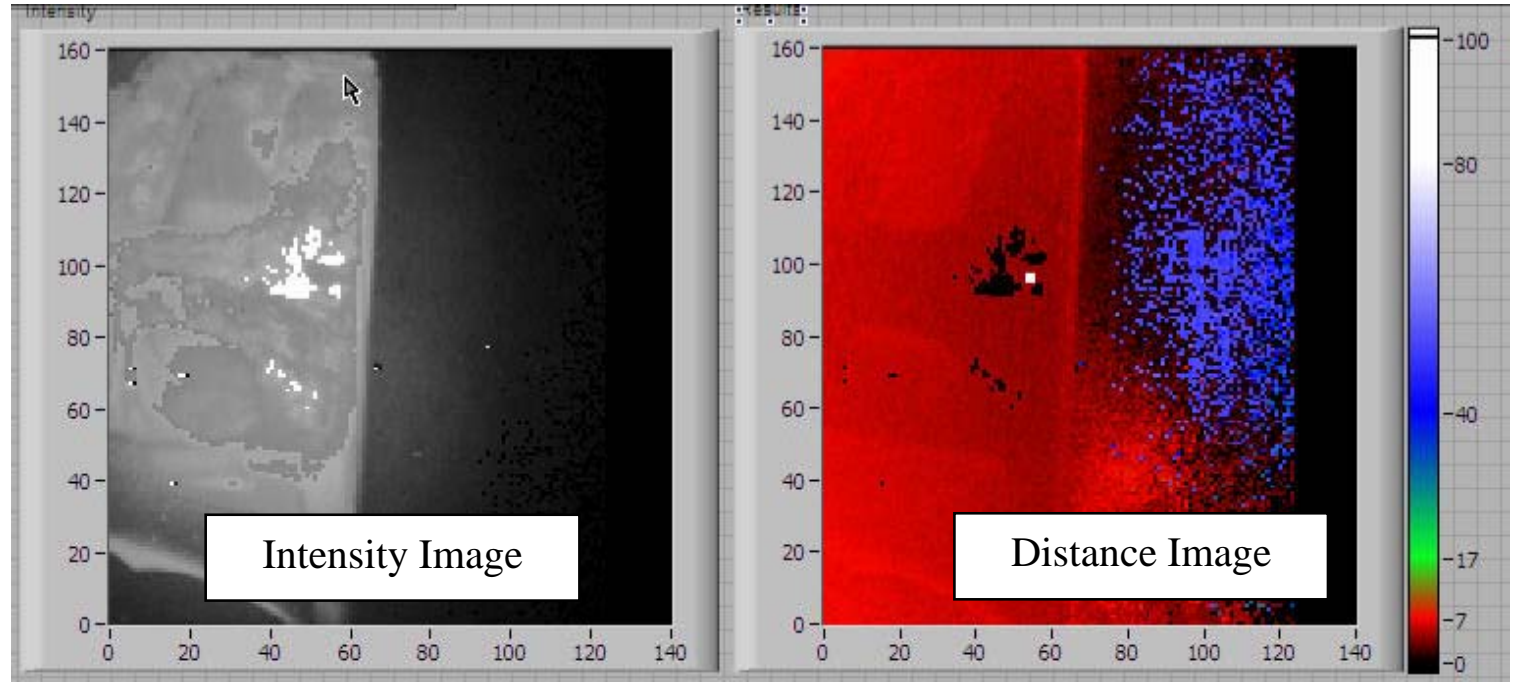

Figure 10 shows a typical image from over five meters. This range approaches the camera's limit and the intensity response is negligible. However the distance image

$\overline{\text { HiCASS Target Tracking Sensor Study }}$ 
shows a vaguely recognizable form. Large amounts of ground clutter noise is evident. Similarly the reflections from the container itself fade as the distance and incidence angle increase. However, due to the large amount of data in a range camera image, there is sufficient data for the algorithm to detect the horizontal edges and the container corner.

Figure 10. Corner Detection at $5 \mathrm{~m}$

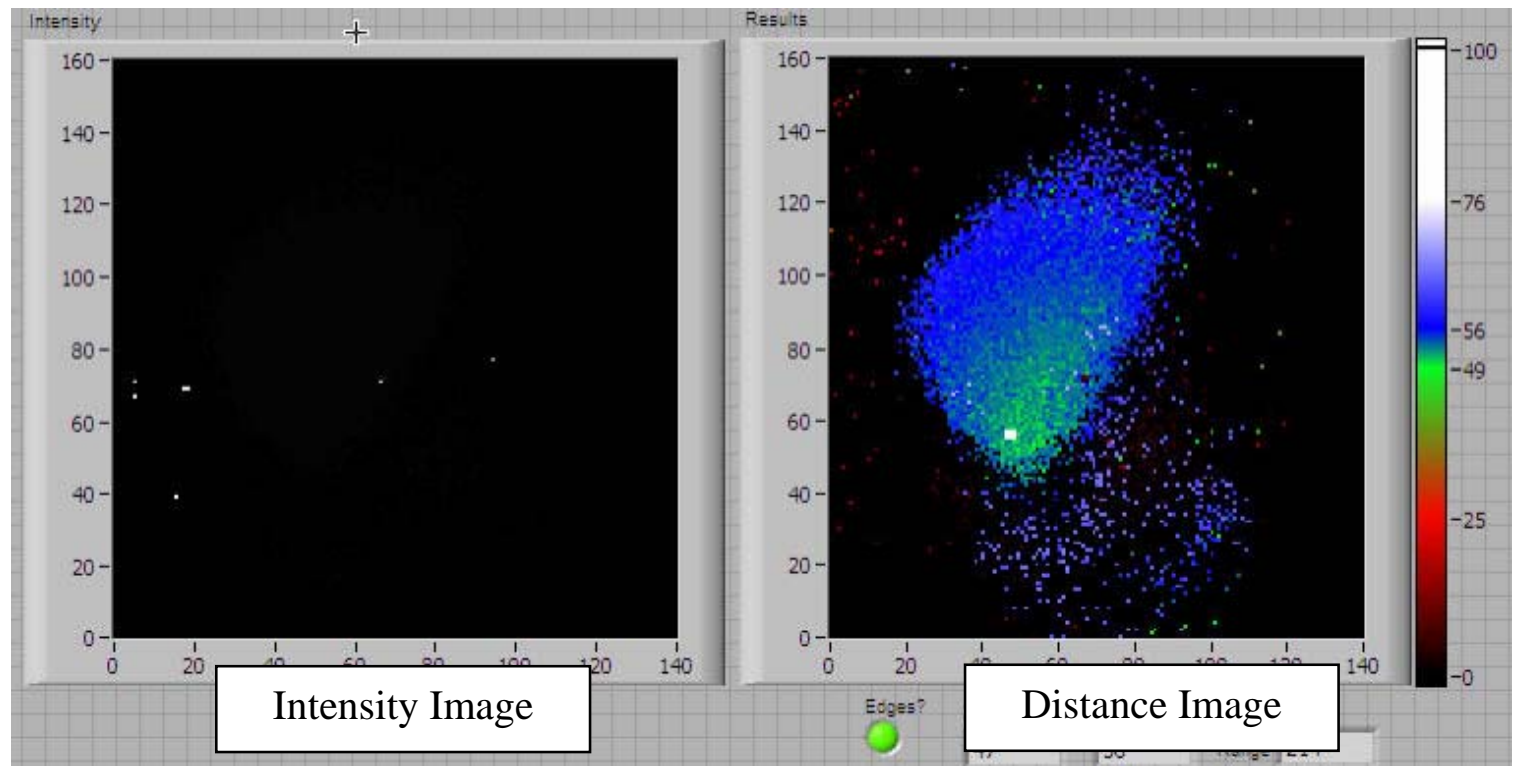

The intended target is the corner fitting of an ISO container as viewed from the opposite octant. We evaluate the precision of the computed position through the standard deviation of readings from 49 sets of data taken from numerous offsets. The experimenters compute the standard deviation through the deviation score of the readings. The standard deviation is $1.9 \mathrm{~cm}$ and the prediction interval for target detection is $5.4 \mathrm{~cm}$. This precision meets the HiCASS requirements.

\subsubsection{Timeliness, Impact, and Readiness}

The 3-D range camera generates 20 images per second. The range camera uses nonilluminated images to identify ambient noise. Users may increase the frame rate by taking fewer non-illuminated images. However, images at higher frame rates will have more noise in dynamic environments. The default rate of 20 images per second meets the HiCASS requirement.

The 3-D range camera has minimal impact on shipboard procedures but is very restrictive for HiCASS with the current capabilities. The daylight restriction makes the current 3-D range camera unacceptable for HiCASS. We anticipate optical and focal array improvements will correct this problem.

The SwissRanger 2 is a pre-production prototype. The manufacturer uses forced convection to cool the electronics. The package is unsealed and suitable only for interior office-type environments. The 3-D camera requires significant re-packaging to meet HiCASS requirements.

HiCASS Target Tracking Sensor Study 


\subsection{Panning Line Scan LADAR}

The linearity of the scanning ladar response, for a given target in a given environment, is excellent. However, LADAR accuracy is also a function of environmental conditions. Surface reflectivity, temperature, and humidity are three factors that significantly effect the range data. According to the manufacturer, distance values may vary by up to $6 \mathrm{~cm}$. However, ISO containers have less variable surface reflectivity then the world in general and local calibration may compensate for atmospheric effects. Therefore, the accuracy of the panning line scan ladar is acceptable for HiCASS.

The spreader bar mounted sensor must observe beyond the ISO container in order to track the vessel after the container is acquired or when delivering a container. The Sick LMS reads distances every degree. At $3 \mathrm{~m}$ the Sick LMS measures lateral position within 0.013 times the distance measurement error. The Sick LMS distance readings have a standard deviation of $10 \mathrm{~mm}$ for 100 samples. This measurement produces a distance prediction interval of $2.8 \mathrm{~cm}$ and a position prediction interval (vector sum of distance and displacement) of $2.9 \mathrm{~cm}$. This precision is acceptable for the HiCASS application.

The motor mounted ladar reads an image in 5 seconds. The tilting mirror alternative takes 3.66 s per image in stepping mode and 1.46 s per image in continuous mode. The continuous mode image tends to have less lateral accuracy due to coordination difficulties between the rotating mirror in the Sick LMS and the tilting mirror. All of these times are for low resolution images $\left(1^{\circ} \times 1^{\circ}\right)$. A high resolution image $\left(1 / 4^{\circ} \times 1 / 4^{\circ}\right)$ takes 18.4 seconds. Therefore, the timeliness of the panning line scan ladar is unacceptable for the HiCASS application.

Because the line scan ladar is operated in short range mode, the panning line scan ladar would be mounted on the spreader bar. The device would image the target ISO container during acquisition tracking and an adjacent container during departure tracking. The panning line scan ladar would have no significant impact on the operations of the HiCASS manipulator or the cargo ship's crew.

The Sick LMS and panning actuators are both COTS products and both are available in hardened packages suitable for maritime environments. The HiCASS controller requires software to interpret the images and to coordinate the sensor's action. However, every sensor requires such software. Therefore the Panning Line Scan LADAR hardware is ready for HiCASS.

\subsection{Fanning Laser Tracker}

Arc Second reports exceptional accuracy and precision for the Constellation 3D-I system. The system is fast and, once calibrated, convenient. The system design is suitable for external applications and is currently undergoing sea trials.

Arc Second measures the standard deviation to be $79 \mu \mathrm{m}$ (0.02 in) from 2 seconds of averaged data. Since the Constellation system outputs data at $40 \mathrm{~Hz}$, the 2 seconds represents 80 samples and the system would average two readings in the HiCASS application. The size of the prediction interval is;

HiCASS Target Tracking Sensor Study 


$$
P=79 \mu m \times t(79,0.95) \times \sqrt{1.5}=1.2 \mathrm{~cm}(0.05 \mathrm{in})
$$

Arc Second collected the data in an airplane manufacturing facility [4]. The facility was a large, temperature controlled room with laser station mounts on the walls and ceiling. HiCASS would have neither environmental controls nor as convenient mounting locations. However, the Constellation 3D-I system would meet HiCASS requirements even if the impact of a harsher environment increased the prediction interval by $600 \%$.

Fanning laser tracker receivers are available in fixed position and wireless configurations. The HiCASS application requires wireless receivers to be configured into targets so the position and orientation of the ISO container may be derived. The ship's crew attaches one target to the desired ISO container and another on a nearby container. The HiCASS controller tracks the second target after capturing the desired ISO container. Between loads the crew would move the targets for the next load. The target on the captured container would have to be returned to the cargo ship for reuse. These activities are a major impact on the cargo ship's crew.

The fanning laser tracker is ready for HiCASS. The Constellation 3D-I system is currently undergoing sea trials. Results of these trial will be available within a couple months.

\subsection{Scanning Laser Tracker}

The Tracker II exceeds the HiCASS requirements for accuracy and precision. Automated Precision manufactures the sensor for industrial environments. However the sensor will have major impact on the crew operations and the timeliness may not be sufficient.

The tracker transmitter may be mounted on the micro-manipulator or on the crane base. When mounted on the crane, the micro-manipulator or a crew member may pass between the transmitter and receiver, cause an interruption, and lose tracking. The scanning laser tracker has fewer problems when mounted on the micro-manipulator.

We envision the tracker transmitter to be mounted on the micro-manipulator and the receiver mounted on the ISO container beneath the target container. The sensor tracks the same target upon approach and departure. The sensor follows the same target upon approach and departure. The Tracker II claims an dynamic absolute position 3-D accuracy of $10 \mathrm{ppm}$ (2 sigma). At $3 \mathrm{~m}$ (10 ft), the sensor's standard deviation is $1.5 \mathrm{~cm}$ (.06 in) which yields a prediction interval of $0.4 \mathrm{~cm}$. The manufacturer claims an orientation resolution of 3 arc-seconds. If uncertainty is 10 times the resolution, the additional error for the upper corner fitting is $0.38 \mathrm{~mm}$ (0.015 in). This value is sufficient to track the ISO container for mating without guide fingers.

The Smart Trak seriously impacts the ship operations. Because the Smart Trak system uses an active receiver, the ship's crew must transfer receivers between loads. However, only the sensor requires only one target for both position and orientation and the target weighs about $1.3 \mathrm{~kg}$ (3 lbs). The crew can handle the target.

HiCASS Target Tracking Sensor Study 
The manufacturer claims several thousand readings per second, which exceeds the HiCASS timeliness requirement. However, any interference between the transmitter and receiver breaks the link. The tracker requires several seconds to reacquire the receiver once the link is lost.

\section{Conclusions}

None of the evaluated sensor systems fulfill all of the HiCASS requirements. Error! Reference source not found. shows a comparison between the five alternatives. The table displays four levels of compliance with the HiCASS goals. "®" indicates the sensor fell significantly below the goal and sufficient improvements are unlikely. The symbol " $\odot$ " indicates the sensor did not meet the requirement but improvements are possible.

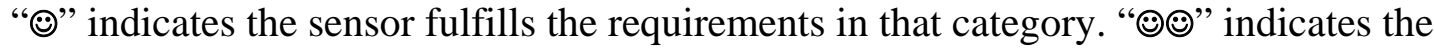
sensor exceeds the requirement. The final row summarizes the desired HiCASS criteria.

Table 2. Comparison Summary

\begin{tabular}{|c|c|c|c|c|c|}
\hline Sensor & Precision & Frequency & Impact & Accuracy & Ready \\
\hline UWB RFID & $\Theta$ & $\theta$ & (ச) & 6 & $\Theta$ \\
\hline TOF Range Camera & (ت) & (ت) & $\ominus$ & (?) & $\ominus$ \\
\hline Panning Line Scan & 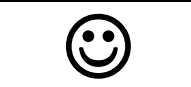 & 6 & 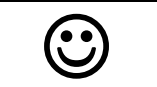 & $(\bullet$ & ( \\
\hline Fanning Tracker & (ن) & $(\bullet)$ & 6 & $(0)$ & ( \\
\hline Scanning Tracker & $(\bullet)$ & $\Theta$ & 6 & $(0)$ & $(\dot{)}$ \\
\hline (i) is & $<7.5 \mathrm{~cm}$ & $20 \mathrm{~Hz}$ & Minor & $\mathrm{r}^{2}>0.99$ & Commercial \\
\hline
\end{tabular}

The UWB alternative does not have sufficient accuracy, precision, frequency, or readiness. The precision and frequency may be improved through modifications to the UWB system. Due to the environmental effects, sufficient improvements to the accuracy are unlikely. The alternative is attractive due to a low impact on the cargo ship's crew and the potential to operate during periods of low visibility.

The precision and timeliness of the 3-D range camera meet the HiCASS requirements. The range camera alternative adds no burdens to the cargo ship's crew. The lens system, and the need to adjust the lens, diminishes the system accuracy. The range cameras are prototypes, are not hardened, and have several inadequacies. The most significant problem is the inability to operate in daylight. The manufacturer identifies several enhancements that may correct the inadequacies.

The panning line scan ladar fulfills all of the HiCASS requirements except timeliness. The panning line scan requires 20 seconds to capture a high resolution image and 5

$\overline{\text { HiCASS Target Tracking Sensor Study }}$ 
seconds for a low resolution image. We include a possible modification in the Recommendations section below.

Laser Trackers have excellent accuracy and precision. We consider two laser trackers. Both of the laser trackers have sufficient timeliness and both are hardened commercial systems. However both use active targets which must be attached to the containers by the cargo ship's crew. The scanning laser tracker suffers from a 3 second re-acquisition time if the transmitter-receiver link is broken.

\section{Recommendations}

Our results justify further investigations into three of the sensor alternatives.

Consideration of the other two may be discontinued. The HiCASS prime contractor may also investigate additional sensors.

\subsection{UWB RFID}

The number of enhancements required to make the UWB usable by HiCASS are substantial. The UWB tracking system should be pursued for HiCASS only if the tag system has been selected by the NTAV program.

\subsection{3-D Range Camera}

3-D Range Cameras are relatively new devices. Although not ready for HiCASS in their current state, the manufacturer acknowledges several improvements which may add sufficient capabilities. These include IR lens designs with coatings to reduce glare and the use of LASER diodes for illumination. The HiCASS program should encourage the manufacturer to make these improvements. If the improvements correct the sensor's deficiency, the 3-D range camera would be an excellent target tracking sensor for HiCASS.

\subsection{Panning Line Scan LADAR}

The Panning Line Scan LADAR meets all of the HiCASS requirements except timeliness. Timeliness improvements are not likely.

\subsection{Fanning Laser Tracker}

The Fanning Laser Tracker fulfills most of the HiCASS requirements. The crew impact cannot be reduced but may be tolerable. We recommend further investigation.

\subsection{Scanning Laser Tracker}

The Scanning Laser Tracker fulfills most of the HiCASS requirements. The crew impact cannot be reduced but may be tolerable. We recommend further investigation.

$\overline{\text { HiCASS Target Tracking Sensor Study }}$ 


\subsection{Vision}

We did not consider vision based sensor systems. Vision based trackers work well in restricted environments. Since ladars have the required accuracy and vision system can track objects, a coordinated vision-ladar system may be able to fulfill the HiCASS requirements.

\section{References}

[1] ONR BAA Number 04-006.

[2] "Precision Asset Locator (PAL650) User’s Guide”, Rev 1.4, Multispectral Solutions, Germantown, MD, Oct 2003.

[3] "User manual: SwissRanger ${ }^{\mathrm{TM}}$ 2", Centre Suisse d'Electronique et de Microtechnique SA, Zurich, Switzerland, Dec 2003.

[4] "Indoor GPS Technology For Metrology", Arc Second White paper \#071502, Arc Second, Inc., Dulles, VA, 2002.

[5] "Scanning Laser Tracker"

[6] Fontana, Robert J. and Gunderson Steven J., "Ultra-Wideband Precision Asset Location System”, 2002 IEEE Conference on Ultra Wideband Systems and Technologies, Baltimore, MD., May 2002.

[7] USAR reference

[8] Fontana, Robert J. et al, “Commercialization of an Ultra-Wideband Precision Asset Location System”, 2002 IEEE Conference on Ultra Wideband Systems and Technologies, Reston, VA, Nov 2003.

[9] Vining, G. Geoffrey, Statistical Methods for Engineers, Brooks/Cole Publishing Company, Pacific Grove, CA 1998, p176.

[10] Dong Hun Shin, et al, Proceedings. 2003 IEEE/RSJ International Conference on Intelligent Robots and Systems, 2003. (IROS 2003)., Oct 2003.

$\overline{\text { HiCASS Target Tracking Sensor Study }}$ 